\title{
An abundance analysis of the single-lined spectroscopic binaries with barium stars-like orbital elements ${ }^{\star}$
}

\section{The spectroscopic data}

\author{
L. Začs ${ }^{1}$, O. Alksnis ${ }^{1}$, F.A. Musaev ${ }^{2}$, I.F. Bikmaev ${ }^{3}$, and G.A. Galazutdinov ${ }^{2}$ \\ 1 Ventspils International Radio Astronomy Center, Akadēmijas laukums 1, LV-1050 Rīga, Latvia \\ e-mail: zacs@latnet.lv \\ 2 Special Astrophysical Observatory, 357147 Nizhny Arkhyz, Russia \\ 3 Department of Astronomy, Kazan State University, 420008 Kazan, Russia
}

Received December 7, 1998; accepted January 21, 1999

\begin{abstract}
High-resolution spectroscopic observations are presented for 19 single-lined binaries (giants and dwarfs) with orbital elements and mass functions similar to those of barium stars, two radial velocity non-variable barium stars, and two standards. All binary systems contain an unseen low-mass component, presumably, a white dwarf. The equivalent widths and oscillator strengths are given for all measured spectral lines.
\end{abstract}

Key words: binaries: spectroscopic — stars: chemically peculiar — stars: abundances

\section{Introduction}

During the years 1993-94 the observation of a large sample of single-lined spectroscopic binaries with barium stars like orbital elements and unseen companions have been carried out. The program stars were selected with the aim to define a sample of binary systems with orbital parameters similar to those of barium stars but for a range of orbital periods, mass function and eccentricity. The purpose of this project is to obtain a well represented abundance pattern for long period binaries to compare these abundances with solar ones and to analyze the influence of the unseen companion on the atmosphere of the primary star to understand better the sufficient conditions for the formation of barium stars. This paper presents the spectroscopic data analyzed in Paper I (Začs et al. 1997).

Send offprint requests to: L. Začs

* Tables 2 and 3 are only available in electronic form at the CDS via anonymous ftp to cdsarc.u-strasbg.fr (130.79.128.5) or via http://cdsweb.u-strasbg.fr/Abstract.html
Table 1. A journal of observations. The number, identification, visual magnitude, date of each exposure, the telescope, spectrograph, and wavelength range are given

\begin{tabular}{rrlrlll}
\hline No. & HD & $V$ & Date & Tel. & Spectr. & Sp. range $(\AA)$ \\
\hline 1 & 6582 & 5.15 & $2-12-93$ & $1 \mathrm{~m}$ & CEGS & $4400-6900$ \\
2 & 13530 & 5.31 & $2-12-93$ & $1 \mathrm{~m}$ & CEGS & $4400-6900$ \\
3 & 21754 & 4.10 & $29-11-93$ & $1 \mathrm{~m}$ & CEGS & $4400-6900$ \\
4 & 61421 & 0.37 & $20-01-94$ & $1 \mathrm{~m}$ & CEGS & $4400-6900$ \\
5 & 76943 & 3.97 & $3-12-93$ & $1 \mathrm{~m}$ & CEGS & $4400-6900$ \\
6 & 113226 & 2.83 & $19-05-94$ & $1 \mathrm{~m}$ & CEGS & $4400-6900$ \\
7 & 116594 & 6.44 & $26-05-93$ & $6 \mathrm{~m}$ & LYNX & $5000-7200$ \\
8 & 119185 & 8.89 & $27-05-94$ & $6 \mathrm{~m}$ & LYNX & $5000-7200$ \\
9 & 120539 & 4.91 & $20-05-94$ & $1 \mathrm{~m}$ & CEGS & $4400-6900$ \\
10 & 121370 & 2.68 & $19-05-94$ & $1 \mathrm{~m}$ & CEGS & $4400-6900$ \\
11 & 130255 & 8.86 & $25-05-94$ & $6 \mathrm{~m}$ & LYNX & $5000-7200$ \\
12 & 155410 & 5.08 & $5-09-93$ & $1 \mathrm{~m}$ & CEGS & $4400-6900$ \\
13 & 160538 & 6.55 & $21-08-94$ & $6 \mathrm{~m}$ & LYNX & $5000-7200$ \\
14 & 166478 & 7.97 & $22-05-93$ & $6 \mathrm{~m}$ & LYNX & $5000-7200$ \\
15 & 168532 & 5.27 & $4-09-93$ & $1 \mathrm{~m}$ & CEGS & $4400-6900$ \\
16 & 170737 & 8.08 & $9-08-94$ & $6 \mathrm{~m}$ & LYNX & $5000-7200$ \\
17 & 176155 & 5.38 & $6-09-93$ & $1 \mathrm{~m}$ & CEGS & $4400-6900$ \\
18 & 176524 & 4.82 & $1-09-93$ & $1 \mathrm{~m}$ & CEGS & $4400-6900$ \\
19 & 179558 & 7.94 & $11-08-94$ & $6 \mathrm{~m}$ & LYNX & $5000-7200$ \\
20 & 181602 & 7.55 & $11-08-94$ & $6 \mathrm{~m}$ & LYNX & $5000-7200$ \\
21 & 204934 & 8.7 & $9-08-94$ & $6 \mathrm{~m}$ & LYNX & $5000-7200$ \\
\hline
\end{tabular}

\section{Observations}

High resolution spectra of the programm stars were obtained with the $6 \mathrm{~m}$ and $1 \mathrm{~m}$ Telescopes at the Special Astrophysical Observatory (SAO) using the Nasmyth echelle spectrometer LYNX (Panchuk et al. 1993) and coude-echelle spectrometer CEGS (Musaev 1993) equipped with the $580 \times 530$ pixels CCD detectors. Table 1 provides a journal of all observations. Apparent magnitudes are from Hoffleit (1982). 


\section{The spectroscopic data}

The reduction and interpretation of the spectra have been explained in detail in Paper I. In the Tables 2 and 3, we give the list of lines used in the abundance analysis, the atomic oscillator strengths (log $g f)$, and measured equivalent widths (in $\mathrm{m} \AA$ ) for each star. The equivalent widths were measured by interactively fitting single Gaussian functions to the line profiles. The atomic oscillator strengths were taken from Luck \& Bond (1985); Thevenin $(1989,1990)$ and Gurtovenko \& Kostik (1989).

Acknowledgements. This research has been supported (LZ) in part by the ESO C\&EE Programme, the Latvian Scientific Council, and by the Russian Foundation of Fundamental researches (FAM, IFB). LZ thanks the staff of the $6 \mathrm{~m}$ telescope for their assistance with the acquisition of the data.

\section{References}

Galazutdinov G.A., 1992, Spec. Astr. Obs. prepr., 92

Gurtuvenko E.A., Kostik R.I., 1989, Frauenhofer spectrum and the system of the sun oscillator strengths, Naukova Dumka, Kiev

Hoffleit D., 1982, The Yale bright star catalogue, Yale University, New Haven

Luck R.E., Bond H.E., 1985, ApJS 59, 249

Musaev F.A., 1993, Pisma v Astron. Zh. 19, 776

Panchuk V.E., Klochkova V.G., Galazutdinov G.A., Ryadchenko V.P., Chentsov E.L., 1993, Pisma v Astron. Zh. 19, 1061

Thevenin F., 1989, A\&AS 77, 137

Thevenin F., 1988, A\&AS 82, 179

Začs L., Musaev F.A., Bikmaev I.F., Alksnis O., 1997, A\&AS 122,31 